\author{
Piotr Sorbet \\ Uniwersytet Marii Curie-Skłodowskiej \\ lepierre@o2.pl
}

\title{
El vesre: un mecanismo de creación léxica
}

\section{Resumen:}

En esta investigación pretendemos, por un lado, continuar y completar el estudio empezado en otras contribuciones y, por otro, presentar el vesre desde una perspectiva global y para ello queremos situarlo entre los restantes mecanismos de creación léxica de los que dispone la lengua española. Para este fin, nos apoyamos en los datos extraídos de diversas fuentes lexicográficas tanto de carácter general como de carácter contrastivo (español europeo $\neq$ español americano).

Palabras clave: vesre, lunfardo, español de América, creación léxica, morfología, cadena lexicogénica

\section{Abstract:}

\section{Vesre: a Mechanism of Lexical Creation}

In this research we intend, on the one hand, to continue and complete the study started in other papers, and on the other, to present vesre from a global perspective. For this reason, we want to place it among other mechanisms of lexical creation available in the Spanish language. To this end, we extract data from various lexicographic sources of general character as well as of contrastive type (European Spanish $\neq$ American Spanish).

Keywords: vesre, lunfardo, American Spanish, lexical creation, morphology, lexicogenic chain 


\section{Introducción}

No en todas las familias lingüísticas existen las mismas herramientas para acuñar vocablos nuevos. Algunas de ellas son más comunes en unas lenguas que en otras. A veces un mecanismo puede ser más rentable en un idioma que en otro y carecer de ciertas limitaciones (fonotácticas, morfológicas, diatópicas, etc.), mientras que en otro puede ser inexistente o caracterizarse por alguna restricción de mayor o menor alcance.

En este artículo, en primer lugar, queremos ocuparnos de algunos de los mecanismos de creación léxica que se suelen indicar tradicionalmente como productivos en español. Sin embargo, debemos subrayar que las descripciones que vamos a presentar no pretenden ser exhaustivas, sino que tienen como fin resaltar algunas de las peculiaridades de estos mecanismos, lo que nos permitirá posteriormente demostrar la necesidad de incorporar, en los estudios morfológicos del español, un nuevo procedimiento de creación léxica, esto es, el vesre. En segundo lugar, señalaremos las características principales del vesre; asimismo, lo cotejaremos con otros mecanismos de creación de nuevas palabras, remarcando, simultáneamente, tanto sus similitudes como sus diferencias.

\section{Principales mecanismos de creación léxica en español}

Una de las herramientas principales del enriquecimiento del vocabulario español y de las lenguas indoeuropeas es la derivación. Esta consiste, grosso modo, en la creación de un vocablo nuevo por la adjunción de algún afijo como resultado de la cual se obtiene un derivado o una palabra derivada. A pesar de su gran rendimiento, en español no existe uniformidad en lo referente a la descripción de algunos de los subtipos de la derivación. En efecto, es posible destacar dos puntos de vista que, en cierta medida, resultan contradictorios. Según el primero, la derivación puede realizarse principalmente de dos maneras: por un lado, por prefijación; y, por otro, por sufijación (Mańczak, 1989: 118). Conforme al segundo, para poder hablar de derivación 
debe intervenir un sufijo, mientras que cuando a la raíz o al lexema se agrega algún elemento antepuesto, dependiendo del estatus gramatical de este último (prefijo o prefijoide ${ }^{1}$ ), se debe hablar, respectivamente, de derivación o de composición (Černý, 2000: 45). Sin embargo, los prefijos, a diferencia de los sufijos, no cambian, normalmente, la clase de palabra ${ }^{2}$ (Gómez Torrego, 2002: 19) y, por lo general, tampoco modifican el acento del lexema básico (Černý, 2000: 45). Sea como fuere, la derivación es la adición o supresión de un elemento inseparable (el afijo) que depende de otro (la raíz). Esto distingue la derivación de la composición, en la cual el elemento añadido tiene autonomía. De ahí que en la composición las dos o más raíces, bases o palabras puedan funcionar independientemente, de vez en cuando con alguna variación, como en sacacorchos, boquiabierto, correveidile (Seco, 1991: 209; Gómez Torrego, 2002: 17; Alvar Ezquerra, 2006 [1994]: 50).

Ahora bien, tanto la derivación como la composición son mecanismos de creación léxica que presentan numerosas limitaciones. Estas últimas, en primer lugar, pueden concernir a su rentabilidad. Así, entre los sufijos que forman nombres de acción distinguimos, entre otros, -anza (matanza, venganza) y -ción (tasación, clasificación). Este último, apoyado por los lenguajes especializados, es crecientemente productivo (Alvar Ezquerra, 2006 [1994]: 57); en cambio, el otro parece mucho menos rentable en la lengua actual.

En segundo lugar, los sufijos pueden presentar diferencias dialectales de varios tipos. Ciertos sufijos pueden ser característicos de alguna zona, como en el caso de los afijos diminutivos: -chu, -cho. Estos se emplean, en el País Vasco y en sus alrededores, con nombres de personas y con algunos apelativos masculinos, así como femeninos: Pedrochu (Pedro), Javiercho (Javier), Carmenchu (Carmen), niñachu (niña) (Bajo Pérez, 1997: 52).

1 Algunas de las formas que son tratadas por algunos autores como prefijoides a veces reciben otras denominaciones, tales como raíces prefijas, seudoprefijos, afijoides, seudoafijos, elementos prefijos (Černý, 2000: 45; Seco, 1991: 214; Quilis, 1994 [1989]: 476; Alvar Ezquerra, 2006 [1994]: 51; DUE).

2 Hoy en día, ciertos morfemas prefijales (anti-, multi-) tienen la posibilidad de cambiar la clase de palabra: antirrobo $(\leftarrow$ robo $)$, multiuso $(\leftarrow u s o)$. 
En tercer lugar, si añadimos determinados sufijos a un lexema se puede acuñar un derivado que, dependiendo de la zona, tiene diferentes sentidos. Así, por ejemplo, en Argentina, el sustantivo frutilla, creado mediante derivación sufijal diminutiva, significa fresa, de manera que arg. mermelada de frutilla es sinónimo de esp. pen. mermelada de fresa (Nowikow, 2010: 425); en España, en cambio, el derivado en cuestión designa cualquier fruta de menor tamaño.

En cuarto lugar, en función de la variante dialectal, la sufijación puede realizarse a través de morfemas diferentes: barro $\rightarrow$ esp. pen. barrizal = esp. arg. barrial 'sitio encharcado, cubierto de barro' (Nowikow, 2009: 219; DUE)³.

En quinto lugar, algunos sufijos parecen más productivos en ciertas regiones que en otras o por lo menos sirven para crear derivados existentes solo en algunas zonas de habla hispana. Así,-aje, que es un galicismo ${ }^{4}$, se une en República Dominicana, Venezuela a negro, y en Argentina, Paraguay, Uruguay a hembra para crear, respectivamente, negraje 'conjunto de personas de raza negra' y hembraje 'conjunto o grupo de mujeres' (DASALE). Nótese que el significado colectivo de -aje no se restringe a las variantes que acabamos de indicar. Sin embargo, su rendimiento varía en función de la variante dialectal, es decir, el afijo en cuestión se somete a restricciones diatópico-productivas.

En sexto lugar, las limitaciones pueden concernir a las posibilidades combinatorias de los elementos que constituyen la palabra. En efecto, la forma del sufijo a menudo ha de adaptarse a la raíz y/o viceversa. Así -miento toma la forma -amiento si el infinitivo del verbo al que se añade termina en -ar (alargar $\rightarrow$ alargamiento), y la forma -imiento si el infinitivo del verbo termina en -er (mantener $\rightarrow$ mantenimiento) o en -ir (sufrir $\rightarrow$ sufrimiento) (Seco, 1991: 207). Queda así patente una serie de restricciones morfológicas.

${ }^{3}$ El sufijo -al es muy productivo en Hispanoamérica: bambudal, bananal, yerbal, etc.(Kany, 1969: 91-92).

${ }^{4}$ Además de funcionar como sufijo,-aje $(<$ fr. -age) también constituye una parte de los siguientes galicismos: kilometraje ( $<$ fr. kilometrage), reportaje $(<$ fr. reportage), aterrizaje ( $<$ fr. $)$, garaje $(<$ fr. garage), marcaje $(<$ fr. marcage), cronometraje $(<\mathrm{fr}$. $)$, pilotaje $(<\mathrm{fr}$. pilotaje), etc. 
La adverbialización de los adjetivos mediante el sufijo -mente (Quilis, 1994 [1989]: 467) , con la excepción de lo que ocurre en el lenguaje poético, no suele aceptar los adjetivos de color (*azulmente, * rojamente), pero sí los que expresan los grados de luminosidad (claramente, luminosamente, oscuramente) (NGLE, 2010: 150). Se trata en ese caso de una restricción semántica. Observemos que las restricciones pueden variar de un idioma a otro. Así, en rumano el mismo sufijo -mente se añade a los adjetivos que terminan en -al: actual $(\rightarrow$ actualmente) o real ( $\rightarrow$ realmente): Sunt realmente bolnav. 'Estoy realmente enfermo'. Sin embargo, en la mayoría de las situaciones restantes, la adjunción del afijo en cuestión parece bastante limitada ${ }^{6}$ (Mirska-Lasota, 1964: 128). Por lo tanto, en rumano la restricción es más morfológica que en español.

Otra de las restricciones combinatorias podemos ilustrarla sirviéndonos de los compuestos. Entre estos últimos, en español hay muchos que se componen de verbo + sustantivo: abrelatas, aguafiestas, correcalles, tirachinas, sacacorchos, pero, al parecer, son relativamente escasos los compuestos de tipo sustantivo + verbo ${ }^{7}$. En otros idiomas, en cambio, esta combinación es más frecuente (cfr. pol. mrówkojad 'oso hormiguero'8, wodociag 'conducción de agua, tubería', listopad 'noviembre', winobranie 'vendimia') ${ }^{9}$. A nuestro juicio, esto puede

${ }^{5}$ Los adverbios que terminan en -mente son compuestos (Gómez Torrego, 2002: 27).

${ }^{6}$ En rumano los adverbios tienen normalmente las mismas formas que los adjetivos. Sin embargo, existen ciertas excepciones: así, los adjetivos terminados en -esc (cf. pol. -ski) forman adverbios en -ește: v. gr., omenesc 'humano' $\rightarrow$ omenește 'humanamente' (Mirska-Lasota, 1964: 128).

${ }^{7}$ Las pocas excepciones las constituyen, quizás, los verbos: maniatar, perniquebrar.

${ }^{8}$ En este caso, se trata de una voz creada por disyunción (Alvar Ezquerra, 2006 [1994]: 26-28).

${ }^{9}$ Los participios acabados en -ante, -ente, -inte (hablante, estudiante) en el español de hoy en día, a pesar de mantener una relación con los verbos de los que derivan, son formas lexicalizadas (Mikołajczyk, 2014: 155); por ende, funcionan como sustantivos. En consecuencia, los compuestos del tipo de hispanohablante están, desde un punto de vista sincrónico, constituidos por dos sustantivos. 
deberse al carácter sintáctico del español, que se identifica como una lengua de tipo SVO.

Asimismo, como en el caso de los derivados, existen numerosos compuestos restringidos a alguna(s) zona(s). Así, cuidacoches 'persona que vigila automóviles estacionados en la vía pública y que recibe una propina por ello' y cuidaculos 'protector de la castidad de una mujer' son, según indican diversas fuentes lexicográficas (DASALE, DLE, DIEA, DELOC, entre otros), argentinismos.

El siguiente recurso que permite enriquecer el vocabulario es el préstamo lingüístico, proceso que implica necesariamente ciertos aspectos vinculados a la adaptación ortográfica, fonética, morfológica, sintáctica, semántica, etc. Así, es importante recordar que, muy a menudo, los vocablos que entran en un idioma experimentan cambios de diversos tipos, como se puede apreciar en los siguientes galicismos: fr. chalet $>$ esp. chalé, fr. capot $>$ esp. capó (apócopes), fr. sirop $>$ esp. sirope (paragoge adaptativa ${ }^{10}$ ), fr. coffre $>$ esp. cofre (reducción ortográfica), fr. reportage $>$ esp. reportaje (sustitución grafemática), etc. Según se aprecia, en el proceso de tomar prestado un vocablo se producen modificaciones de diferentes caracteres, que se deben, a veces, a restricciones fonotácticas, ortográficas, morfológicas o de otro tipo, que varían de unos idiomas a otros. A modo de ejemplo, en polaco el primer galicismo que hemos indicado arriba, que tiene la forma szalet 'excusado público', se diferencia del galicismo español a nivel ortográfico, semántico, así como morfológico.

Otro mecanismo de creación léxica es la eponimia, que consiste, a grandes rasgos, en la conversión de un nombre propio en un nombre común. Su rentabilidad depende del contexto social, político, histórico, etc. Así, son voces epónimas los nombres de movimientos políticos de distintos países: esp. pen. zapaterismo o esp. arg. kirschnerismo, cuyas denominaciones provienen respectivamente de José Luis Rodríguez Zapatero y Néstor Kirchner y Cristina Fernández de Kirchner. Debemos tener presente que los dos vocablos tienen ciertas restricciones

${ }^{10}$ Para lo que se refiere a la clasificación de los cambios paragógicos en las lenguas románicas, $c f r$. Sorbet, 2015. 
diatópicas, así como diacrónicas, ya que, sin duda, con el paso del tiempo se usarán con menos frecuencia para convertirse en palabras históricas.

La siguiente herramienta que permite acuñar vocablos nuevos la constituyen los acortamientos de diversos tipos, por ejemplo, el abreviamiento o truncamiento (Álvar Ezquerra, 2006 [1994]: 45). Este consiste en "la reducción del cuerpo fónico de una palabra", en la mayoría de las situaciones por apócope (metropolitano $\rightarrow$ metro) o por aféresis (autobuis $\rightarrow$ bus). Este mecanismo es sumamente rentable, ya que permite transmitir una información más rápidamente y es conforme a la ley del menor esfuerzo ${ }^{11}$. Por estos motivos, parece que es universal y se registra en todas las lenguas del mundo: ing. gymnastics $\rightarrow$ gym, fr. pneumatique $\rightarrow$ pneu, jap. スーパーマーケット (sūpāmāketto) $\rightarrow$ スーパー (sūpā). Sin embargo, notemos que el acortamiento no se produce de la misma manera en diferentes idiomas. Así, este puede realizarse en unas lenguas por aféresis y en otras por apócope. Por ejemplo, el equivalente de matemáticas se abrevia coloquialmente en checo suprimiendo el primer elemento (matika $\leftarrow$ matematika); en cambio, en pol. lo que se elimina es el segundo elemento (matma $\leftarrow$ matematyka) (Tarajło-Lipowska, 2011: 12).

Ya hemos demostrado que ciertos recursos, por muy variados que sean sus posibles desarrollos, son más comunes que otros. Sin embargo, también hemos afirmado que hay lenguas que carecen de procedimientos que, en cambio, son muy rentables en otras. A título de ejemplo, podemos indicar el caso de la conversión sustantivo $\rightarrow$ verbo, que en la lengua española es inexistente, mientras que en inglés es bastante frecuente. Esto se debe a que en español para crear un infinitivo se necesita la terminación infinitiva $-r$, lo que significa que esta transformación tiene un carácter morfológico; en contrapartida, en inglés este requisito no es obligatorio y por ello es un proceso más bien sintáctico (Mikołajczyk, 2014: 154, 158).

${ }^{11}$ La formación de acortamientos puede ser interpretada como el resultado de la ley de desarrollo irregular debido a la frecuencia de Mańczak (1989: 14). 
Todas las formas que hemos examinado hasta ahora nos permiten sostener que en los mecanismos de creación léxica existen numerosas restricciones diatópicas, semánticas, productivas, morfológicas (o combinatorias), etc., que dependiendo, por ejemplo, del idioma, pueden aplicarse de diferentes maneras.

La lista de los mecanismos que acabamos de describir concisamente no está completa. No hemos hablado de la sinapsia (Alvar Ezquerra, 2006 [1994]: 23), la parasíntesis (Alvar Ezquerra, 2006 [1994]: 65; Černý, 2000: 58; Gómez Torrego, 2002: 18), las creaciones onomatopéyicas (Alvar Ezquerra, 2006 [1994]: 15), el reanálisis (Nowikow, 2009: 222) y algunos otros más. Sin embargo, la relación, que entendemos suficiente para la exposición de las características principales del vesre, nos permitirá compararlo, asimismo, con los recursos de formación de palabras que hemos presentado arriba.

\section{Vesre: características generales}

Como ya hemos mencionado supra, en este trabajo queremos continuar con la investigación que hemos emprendido en otras contribuciones, situando en este caso el vesre entre los demás recursos de creación léxica. Sin embargo, antes de pasar a ello, creemos que es imprescindible resumir someramente las observaciones que ya hemos realizado en otras ocasiones (Sorbet, 2014, 2016a, 2016b), sobre todo porque en las diversas fuentes es posible encontrar informaciones, en cierta medida, contradictorias acerca del carácter del vesre.

En primer lugar, hay que hacer hincapié en que no es un fenómeno lingüístico limitado exclusivamente a la zona porteña de Argentina, como se podría inferir apoyándose en ciertos diccionarios o trabajos lingüísticos (Sorbet, 2016a, 2016b). En efecto, los vesreísmos (cfr. infra) se registran también en países como, entre otros, Bolivia, Chile, Colombia, Costa Rica, Ecuador, Honduras, Nicaragua, Panamá, Perú, Paraguay, Puerto Rico o Uruguay (Sorbet, 2016a, 2016b: 145-150).

En segundo lugar, aunque en ciertas ocasiones se ha sostenido que los vocablos vésricos ( $c f r$. infra) se han creado por metátesis, opinamos que no es posible identificar el vesre con la metátesis. Esta 
no tiene como objetivo esencial la creación de una palabra nueva, a pesar de que debemos reconocer que en algunas situaciones este es su resultado. En la metátesis, lo que cambia de posición son preponderantemente los sonidos simples: lat. percontāri $\rightarrow$ preguntar (cfr. port. perguntar), lat. pericŭlum $\rightarrow$ esp. peligro (cfr. fr. péril), lat. parabola $\rightarrow$ palabra (cfr. fr. parabole) (cfr. tb. Sorbet, 2016b: 143) ${ }^{12}$. En el vesre el reordenamiento no concierne solo a sonidos simples sino también a sílabas enteras (Sorbet, 2016b: 143). Además, su objetivo principal, como en el caso de los demás mecanismos de creación léxica, es acuñar una forma (voz) nueva. Esta última se puede forjar con fines poéticos, humorísticos, crípticos, eufemísticos, disfemísticos o identificadores de alguna generación (cfr. Sorbet 2016b: 149; Sorbet, 2016c: 162). Asimismo, hay que subrayar que la metátesis no es un fenómeno que se produzca conscientemente de una manera regular. El vesre no solo es un recurso lingüístico que se utiliza conscientemente, sino que también es, desde luego, regular. En efecto, las voces creadas en el marco del vesre se forman de acuerdo con algunas fórmulas que se repiten continuamente. A fin de ilustrarlo podemos recoger vesreísmos lunfardescos que se generan a base de voces trisilábicas de las siguientes maneras:

$\begin{array}{lll}\text { a) } \# 321 \# & \text { pelota }\{\text { pe-lo-ta }\} \rightarrow \text { talope; } \\ \text { b) } \# 312 \# & \text { boliche }\{\text { bo-li-che } \rightarrow \text { cheboli; } \\ \text { c) } \# 213 \# & \text { trabajo }\{\text { tra-ba-jo } \rightarrow \text { batrajo; } \\ \text { d) } \# 132 \# & \text { conmigo }\{\text { con-mi-go } \rightarrow \text { congomi; } \\ & \text { e) } \# 231 \# & \text { patrona }\{\text { pa-tro-na } \rightarrow \rightarrow \text { tronapa. }\end{array}$

Naturalmente, dichas fórmulas no tienen el mismo rendimiento. La primera de ellas es más común, mientras que la última parece mucho menos frecuente. De ahí que opinemos que sobre ellas se imponen ciertas restricciones de índole morfológica o de productividad.

${ }^{12}$ Según algunos autores, la metátesis concierne a dos sonidos que no están contiguos, mientras que cuando se trata de dos sonidos vecinos se debe hablar de inversión (Quilis, 2010 [1997]). 
Además, es crucial subrayar que una voz puede vesrearse mediante más de una fórmula: trabajo $\rightarrow$ jotraba (b) o batrajo (c), querida $\rightarrow$ darique (a) o daqueri (b) (Sorbet, 2016a). En ocasiones las diferencias entre las fórmulas que se utilizan distinguen, según las fuentes lexicográficas, las variantes dialectales (Sorbet, 2016b: 146), lo que se podría comparar con el uso de distintos sufijos con el fin de crear derivados con el mismo significado, pero con formas discrepantes (cfr. supra).

Conforme a las premisas que ya hemos expuesto en otras ocasiones (Sorbet, 2014, 2016a, 2016b), estamos convencidos de que en el vesre se deben distinguir dos términos: la voz vesreable y el vesreísmo o voz vesreada. El primero designa la palabra susceptible de ser vesreada; en cambio, el segundo es cualquier palabra que ha sido previamente vesreada. Asimismo, el proceso realizado de acuerdo con las fórmulas indicadas arriba, cuyo resultado es la creación de una forma nueva, es el vesreamiento.

El hecho de que existan ciertas fórmulas que se repiten frecuentemente y que no presentan ninguna modificación suplementaria a nivel morfológico no significa que no haya casos irregulares. En efecto, en el vesreamiento pueden intervenir también otros fenómenos en la creación de una palabra nueva. Nos referimos, entre otros, a las modificaciones: fonéticas (inclusive la prosodia), morfológicas u ortográficas: doctor $\rightarrow$ tordo (apócope), cabaret $\rightarrow$ bareca (síncope), pantalón $\rightarrow$ lompa(s) (aféresis), hotel $\rightarrow$ telo (supresión de la $h$ ), tango $\rightarrow$ gotán (cambio de acentuación), etc. Por consiguiente, es posible sostener que las voces vesreables y las fórmulas básicas, en ciertas circunstancias, deben acomodarse para poder crear un vesreísmo. Esto significa que existen, como en el caso de la derivación, de la composición o del proceso de tomar préstamo, algunas restricciones fonéticas, morfológicas, etc. Observemos, no obstante, que estas son relativamente escasas. E1 vesre, por lo tanto, es un mecanismo de creación léxica cuya productividad es potencialmente muy grande.

Expuestas estas observaciones, ahora ya podemos volver a insistir, presentando un argumento suplementario, en que el vesre no se debería confundir con la metátesis. En efecto, en el vesre esta última, a nuestro modo de ver, puede acompañar como la aféresis, la apócope 
$\mathrm{y}$ otras modificaciones, al proceso del vesreamiento: hembra $\rightarrow$ brame (metátesis simple), macho $\rightarrow$ chamo (metátesis recíproca). Los dos ejemplos confirman, así, las observaciones que ya hemos presentado en otras ocasiones (Sorbet, 2016a, 2016b).

En cuanto a la distribución diatópica, el vesre, desde luego, no se utiliza con la misma frecuencia en todas las regiones de habla hispana. Por ello, creemos que es conveniente hablar de restricciones dialectales. Sin embargo, como ya hemos indicado arriba, los vesreísmos se registran, por lo menos ocasionalmente, en amplias zonas de Hispanoamérica y, en algunos casos, en España (Sorbet, 2016b: 144-145).

Por lo que se refiere al nivel semántico, es necesario recalcar que los vesreísmos no son meras variantes de las voces vesreables de las que proceden, al igual que un derivado no puede ser considerado, por lo general, solo como un sinónimo de la palabra base ya que está última carece del afijo que modifica supuestamente su significado. Los vesreísmos tienen, por tanto, sus propios sentidos. Además, los vesreísmos y los vocablos que han servido para forjarlos pertenecen, con mucha frecuencia, a registros diferentes. Así, en Argentina feca no es un sinónimo de todas las acepciones de café ni pertenece a su mismo registro (Sorbet, 2016a). El vocablo pelpa es, a la vez, un vesreísmo de papel 'pliego, hoja o pedazo de papel', así como del lunfardismo papel 'dosis de cocaína de entre 0,5 y 1 gramo envuelta en un sobrecito o papel' (DELOC). Analógicamente, naerpi tiene, simultáneamente, las acepciones de pierna 'extremidad inferior del hombre' y del sustantivo lunfardesco pierna 'persona dispuesta a prestar compañía' (DELOC). La voz vésrica telo $\leftarrow$ hotel significa 'hotel destinado a citas de amantes' (Sorbet, 2016b: 151). Además, un vesreísmo puede adquirir acepciones diferentes según las variantes (cfr. Sorbet, 2016b: 151), como ocurre también con los demás recursos de formación de palabras (cfr. supra).

A raíz de lo que hemos dicho hasta ahora, afirmamos que el vesre presenta los mismos tipos de restricciones (semánticas, morfológicas, diatópicas) que los demás recursos de formación de palabras.

Finalmente, el vesre constituye un mecanismo de creación léxica plena e independientemente aplicable en el sistema ya que puede 
participar en la cadena lexicogénica, concepto cuyas primeras premisas presentamos a continuación.

\section{Cadena lexicogénica}

Según nuestra perspectiva, para poder hablar de un mecanismo de creación léxica en un idioma son indispensables, entre otros, dos factores.

El primero es la productividad. A nuestro juicio, es cuestionable sostener que en un idioma existe un determinado mecanismo productivo si apenas se pueden indicar palabras acuñadas mediante dicho mecanismo. Así, en español la conversión de tipo sustantivo $\rightarrow$ verbo es morfológicamente imposible, y por ello inexistente, puesto que en este idioma para convertir un sustantivo en un verbo es necesaria la terminación infinitiva; en cambio, el inglés no sufre restricciones de esta índole (cfr. supra).

El segundo factor lo constituye la posibilidad del mecanismo en cuestión de integrarse en la cadena lexicogénica. Creemos que, si después de la aplicación de dicho mecanismo de creación léxica, la voz recién forjada puede verse sometida a otro, es decir, si al mecanismo considerado puede sucederle otro regularmente, independientemente de ciertas restricciones que pesan sobre ellos, se puede afirmar que el primero es productivo y forma parte del sistema lingüístico, y que, por lo tanto, es incorporable a la cadena lexicogénica. Veamos tres ejemplos escogidos arbitrariamente.

El compuesto Hispanoamérica ha dado origen al derivado hispanoamericano (Seco, 1991: 209) y este último ha servido para crear la palabra derivada antihispanoamericano. El galicismo cinematógrafo ( $<$ fr. cinématographe) se ha reducido por truncamiento a la forma cine, que, además de funcionar como un sustantivo independiente, se ha convertido, según la concepción que se adopte ( $c f r$ supra), en un elemento prefijo o en un elemento compositivo de los vocablos cineclub y cineteca (DUE). El sustantivo francés guillotine, voz epónima creada a base del apellido (Joseph) Guillotin, ha pasado al español con la forma guillotina. Esta última se ha transformado, posteriormente, en la palabra base para el verbo denominal guillotinar. Todos los casos 
que acabamos de resumir concisamente son cadenas lexicogénicas que pueden ser más largas o más cortas y que incluso a veces pueden desembocar en un verdadero árbol morfológico (cfr. Černý, 2000: 44). En el último ejemplo que hemos indicado, los mecanismos se han sucedido de la manera siguiente: eponimia (epónimo) $\rightarrow$ proceso de tomar prestado (préstamo) $\rightarrow$ derivación (derivado):

\begin{tabular}{|c|c|c|c|c|c|c|}
\hline \multirow{2}{*}{$\begin{array}{c}\text { Joseph } \\
\text { Guillotin }\end{array}$} & $\rightarrow$ & \multirow{2}{*}{$\begin{array}{c}\text { fr. } \\
\text { guillotine }\end{array}$} & \multirow{2}{*}{$\begin{array}{c}> \\
\text { proceso }\end{array}$} & \multirow{2}{*}{$\begin{array}{c}\text { esp. } \\
\text { guillotina }\end{array}$} & $\rightarrow$ & \multirow{2}{*}{ guillotinar } \\
\hline & \multirow{2}{*}{ eponimia } & & & & \multirow[b]{2}{*}{ derivación } & \\
\hline $\begin{array}{l}\text { nombre } \\
\text { propio }\end{array}$ & & epónimo & $\begin{array}{l}\text { de tomar } \\
\text { prestado }\end{array}$ & préstamo & & derivado \\
\hline
\end{tabular}

Los diferentes mecanismos de creación léxica son, por lo tanto, recíprocamente complementarios y se desarrollan secuencialmente. Valiéndonos de los ejemplos que hemos examinado anteriormente, podemos presentar esquemáticamente también la siguiente cadena lexicogénica:

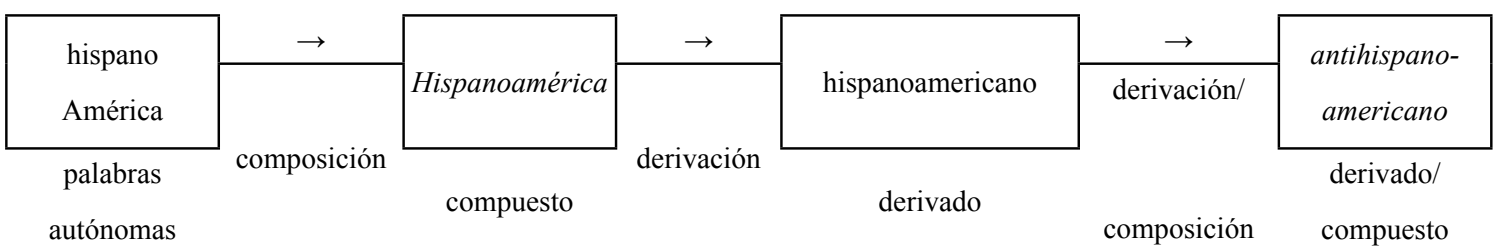

Por lo que se refiere al vesre, este, a pesar de caracterizarse, como los demás mecanismos, por ciertas restricciones, es plenamente aplicable en el sistema de la lengua española. Valgan los siguientes casos: a) El galicismo chofer (en esp. pen. chófer) < fr. chauffeur tras vesrearse genera las formas fercho, fercha, lo que significa que aquí el vesre sucede al préstamo.

b) En Costa Rica, primo, prima devienen mopri y mapri, que después se apocopan, es decir, por truncamiento posibilitan la creación de la forma mop (¿qué mop?) (Sorbet, 2016b: 148).

c) El sustantivo tango tras el vesreamiento se convierte en gotán, y este último, al añadirle el sufijo -ero/a, da origen al adjetivo gotanero/a, que aparece en la letra del tango Nocturnal bandoneón 
de Juan Bautista Devoto. Por lo tanto, la existencia del vesreísmo hace realizable la creación del derivado (devesreíco).

d) En Costa Rica el sustantivo picha se vesrea en chapi, y este último llega a formar parte del compuesto carechapi (Sorbet, 2016b: 148). Como se muestra, los mecanismos de creación léxica se suceden en las cadenas lexicogénicas, respectivamente, de las siguientes maneras: (a) préstamo $\rightarrow$ vesre, (b) vesre $\rightarrow$ truncamiento, (c) vesre $\rightarrow$ derivación, (d) vesre $\rightarrow$ composición.

Debemos subrayar que el vesre es un mecanismo que, como ya hemos visto arriba, tiene sus limitaciones diatópicas, pero este procedimiento no es intrínsecamente español. En efecto, hay muchas lenguas en las que existen mecanismos, con denominaciones muy variadas, de creación léxica sumamente parecidos. A título de ejemplo destaquemos el verlan del francés, el шатровачки del serbo-croata (Ћосић, 2004) o el binaliktad del tagalo y otros más en los que se pueden indicar numerosas palabras creadas por el vesreamiento.

\section{Observaciones finales}

Como acabamos de examinar, el vesre es un mecanismo de creación léxica, lo que confirma las observaciones que hemos presentado en otras contribuciones.

Opinamos que es necesario distinguir el vesre de entre los demás mecanismos de creación léxica, ya que no solo es potencialmente productivo, sino que también es posible indicar numerosas palabras vésricas creadas en múltiples zonas de habla hispana. Además, es plenamente aplicable en el sistema. Su complementariedad con otros procedimientos de formación de palabras, por ejemplo, en las cadenas lexicogénicas, aunque de una manera muy somera, ha sido ejemplificada y demostrada. Al igual que los demás procedimientos de formación de vocablos, tiene sus restricciones diatópicas y morfológicas. En el vesre se producen ciertos fenómenos complementarios (apócope, aféresis, paragoge). A raíz de todas las características que hemos expuesto podemos sostener que hemos conseguido situarlo entre los demás mecanismos de creación léxica. Sin embargo, sería digno de interés 
cotejarlo, en la medida de lo posible, con los recursos semejantes que existen en otras lenguas.

\section{Bibliografía}

ALVAR EZQUERRA, M. (2006 [1994]), La formación de palabras en español, Arco/Libros, Madrid.

Asociación de Academias de la Lengua Española (2010), Diccionario de americanismos, Santillana, Lima. (DASALE)

BAJO PÉREZ, E. (1997), La derivación nominal en español, Arco/Libros, Madrid.

CONDE, Ó. (2004), Diccionario etimológico del lunfardo, Taurus, Buenos Aires. (DELOC)

ČERNÝ, J. (2000), Morfología española, Univerzita Palackého, Olomouc. GÓMEZ TORREGO, L. (2002 [1997]), Gramática didáctica del español, SM, Madrid.

KANY, Ch. (1969), Semántica hispanoamericana, Aguilar, Madrid.

MAŃCZAK, W. (1989), Fonética y morfología histórica del español, Uniwersytet Jagielloński, Kraków.

MIKOŁAJCZYK, S. (2014), "Uma questão sobre a formação de palavras. Caso de conversão ou derivação imprópria no espanhol, inglês e português", en: Pawlik, J., Szałek, J. (red.) Lingüística española en Polonia. Lineas de investigación, Wydawnictwo Naukowe Uniwersytetu im. Adama Mickiewicza, Poznań, pp. 153-158.

MIRSKA-LASOTA, H. (1964), Zwięzła gramatyka języka rumuńskiego, Wiedza Powszechna, Warszawa.

MOLINER, M. (2008), Diccionario de uso del español, Madrid, Gredos, [CD-ROM]. (DUE)

NOWIKOW, W. (2009), "Algunas observaciones sobre los mecanismos de la creación léxica en el español argentino actual”, en: Luque Toro, L. (ed.), Léxico español actual II, Università Ca' Foscari,Venezia, pp. 219-227.

NOWIKOW, W. (2010), "De modificación morfemática a cambios de aplicación y de significado: sobre un neologismo parónimo en el español de 
Argentina”, en: Le Tallec-Lloret, G. (éd.), Vues et contrevues, Collection Libero, Lambert-Lucas, Limoges, pp. 425-430.

PLAGER, F., (coord.) (2008), Diccionario integral del español de la Argentina, Voz Activa, Buenos Aires. (DIEA)

QUILIS, A., ESGUEVA, M., GUTIÉRREZ, M. L., RUIZ-VA, P. (1994 [1989]), Lengua española. Curso de acceso, Editorial Centro de Estudios Ramón Areces, Madrid.

QUILIS, A. (2010 [1997]), Principios de fonología y fonética españolas, Arco/Libros, Madrid.

Real Academia Española y Asociación de Academias de la Lengua Española (2010), Nueva gramática de la lengua española. Manual, Espasa, Madrid. (NGLE)

Real Academia Española (2016), Diccionario de la lengua española, [on-line] http://www.rae.es, 12.12.2016. (DLE)

SECO, M. (1991 [1989]), Gramática esencial del español, Espasa Calpe, Madrid.

SORBET, P. (2014), “Análisis lingüístico del vesre porteño”, Roczniki Humanistyczne, LXII, pp. 123-134.

SORBET, P. (2015), "Próba klasyfikacji zmian paragogicznych w językach romańskich”, en: Lipiński, D., Witczak, K. T. (red.), Badania diachroniczne w Polsce. Pamięci profesora Witolda Stefańskiego (1953-2013), Wydawnictwo Uniwersytetu Łódzkiego, Łódź, pp. 13-22.

SORBET, P. (2016a), “Aproximación a la descripción lingüística del vesre porteño”, en: Wilk-Racięska, J., Szyndler, A., Tatoj, C. (eds.), Relecturas y nuevos horizontes en los estudios hispánicos, vol. 4: Lingüistica y didáctica de la lengua española, Wydawnictwo Uniwersytetu Śląskiego, Katowice, pp. 173-183.

SORBET, P. (2016b), "En torno al tratamiento lexicográfico de los vesreísmos", Itinerarios, 23, pp. 141-153.

SORBET, P. (2016c), "Sobre los etnónimos oficiosos y la disfemización etnonímica: presentación del tema", en: Posturzyńska-Bosko, M., Sorbet, P. (red.), Le tabou comme défi pour la linguistique. El tabú como desafio para la lingüistica, Wydawnictwo Uniwersytetu Marii CurieSkłodowskiej, Lublin, pp. 153-168. 
TARAJŁO-LIPOWSKA, Z. (2000), Kapoan, czyli język na opak. O czeskim dla Polaków, być może mało zaawansowanych, ale mocno zainteresowanych, Wydawnictwo Uniwersytetu Wrocławskiego, Wrocław.

ЋОСИЋ, П. (2004), “Правила жаргонске метатезе”, Језик данас, 19-20, pp. 14-17. 